\title{
FEV $_{1}$ and $M R I$ ventilation defect reversibility in asthma and COPD
}

\author{
To the Editor:
}

The underlying pathophysiological determinants of asthma and COPD are related in complex ways. Importantly however, post-bronchodilator forced expiratory volume in $1 \mathrm{~s}\left(\mathrm{FEV}_{1}\right)$ reversibility may occur in approximately $50 \%$ of COPD patients [1], whilst epidemiological [2] and magnetic resonance imaging (MRI) studies [3] suggest that, in asthma patients, $\mathrm{FEV}_{1}$ reversibility may diminish over time. As compared to patients with asthma or COPD alone, patients with co-existing asthma and COPD report worse clinical outcomes [4] and increased healthcare costs [5] and burden [4, 5].

Hyperpolarised ${ }^{3} \mathrm{He}$ and ${ }^{129} \mathrm{Xe}$ MRI studies have reported clinically relevant post-bronchodilator improvements in MRI ventilation defects in patients with asthma [6] and COPD [7], independent of FEV 1 reversibility. However, post-bronchodilator $\mathrm{FEV}_{1}$ and MRI reversibility have not been rigorously explored together across a wide range of patients with obstructive lung disease. Although their aetiology has not been ascertained, MRI ventilation defects are thought to be related to airway occlusion or remodelling throughout the airway tree, while $\mathrm{FEV}_{1}$ is dominated by the larger airways.

To better understand bronchodilator reversibility in patients with a diagnosis of asthma or COPD, we retrospectively evaluated spirometry and hyperpolarised ${ }^{3} \mathrm{He}$ MRI measurements in participants with asthma (age $\geqslant 40$ years, <1 pack-year smoking history) and COPD (age 60-85 years, >10 pack-years smoking history). We expected that MRI would detect post-bronchodilator reversibility in participants in whom there was negligible post-bronchodilator $\mathrm{FEV}_{1}$ reversibility, thereby providing a small and large airway quantitative functional measurement with more enhanced sensitivity to small airways disease and greater dynamic range than $\mathrm{FEV}_{1}$.

We retrospectively evaluated 58 participants including 45 participants with asthma (20 male and 25 female never-smokers, $54 \pm 8$ years) and 13 with COPD (seven male and six female ex-smokers, $70 \pm 6$ years, $45 \pm 17$ pack-years) who underwent a single 2-h visit consisting of spirometry and MRI before and after inhalation of salbutamol $(4 \times 100 \mu \mathrm{g}$; Teva Novopharm Ltd, Toronto, ON, Canada) using a pressurised metred-dose inhaler and AeroChamberPlus spacer (Trudell Medical International, London, ON, Canada). Spirometry was performed (MedGraphics, St. Paul, MN, USA) and post-bronchodilator reversibility assessed according to guidelines [8]. Participants withheld short-acting $\beta$-agonists for $6 \mathrm{~h}$ and long-acting $\beta$-agonists for $12 \mathrm{~h}$ before their visit. Anatomical proton and hyperpolarised ${ }^{3} \mathrm{He}$ ventilation MRI were acquired after inhalation from functional residual capacity as previously described [9]. We used the minimal clinically important difference (MCID) of $110 \mathrm{~mL} / 2 \%$ [9] as the threshold for a clinically relevant post-bronchodilator change in MRI ventilation defect per cent (VDP) [9].

In asthma, 51\% (23 of 45) of participants were $\mathrm{FEV}_{1}$ reversible, whereas $15 \%$ (two of 13 ) of COPD participants were $\mathrm{FEV}_{1}$ reversible. Shown in figure 1 (bottom panel), COPD and asthma participants were further classified into eight subgroups based on $\mathrm{FEV}_{1}$ and VDP post-bronchodilator reversibility, where blue represents both $\mathrm{FEV}_{1}$ and VDP reversibility, purple represents either $\mathrm{FEV}_{1}$ or VDP reversibility and pink reflects neither $\mathrm{FEV}_{1}$ nor VDP reversibility. 27\% (12 of 45) of asthmatics and 15\% (two of 13) of COPD participants exhibited both $\mathrm{FEV}_{1}$ and VDP reversibility (blue). As shown in pink, in 33\% (15 of 45) of participants with asthma and 62\% of participants with COPD (eight of 13, approximately double the proportion of asthmatics) there was neither $\mathrm{FEV}_{1}$ nor VDP reversibility. Shown in purple, $24 \%$ (11 of 45) of asthmatics and no COPD participants demonstrated $\mathrm{FEV}_{1}$ reversibility in the absence of VDP reversibility, whilst $16 \%$ (seven of 45 ) of asthmatics and $23 \%$ (three of 13) of COPD participants

@ERSpublications

MRI ventilation defect post-bronchodilator reversibility was not always concordant with $\mathrm{FEV}_{1}$ reversibility in asthma and COPD. MRI VDP may provide enhanced sensitivity to small airway response to inhaled medication. http://bit.ly/2qKnwID

Cite this article as: Serajeddini $\mathrm{H}$, Eddy RL, Licskai C, et al. $\mathrm{FEV}_{1}$ and MRI ventilation defect reversibility in asthma and COPD. Eur Respir J 2020; 55: 1901947 [https://doi.org/10.1183/13993003.01947-2019]. 

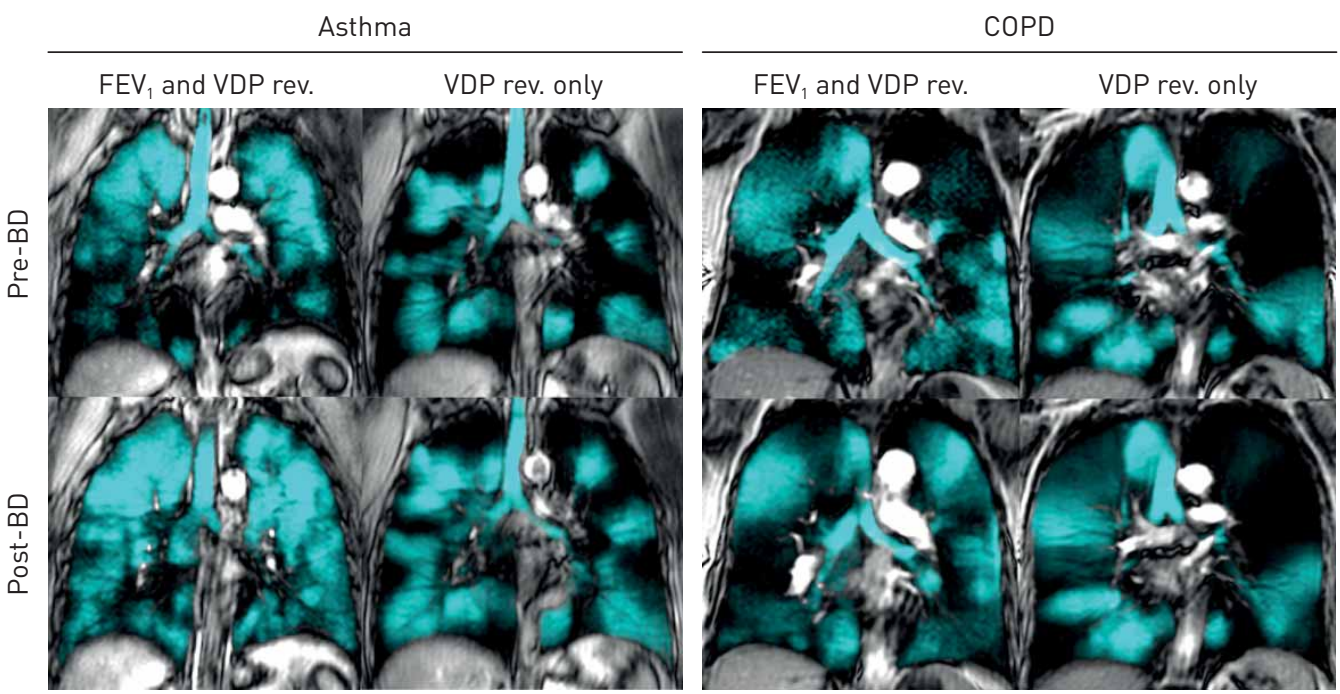

\begin{tabular}{lc}
\hline Asthma $\mathrm{n}=45$ & Pre/PostBD \\
\hline Age years & $54 \pm 8$ \\
Female & $25(56 \%)$ \\
FEV $_{1} \%$ pred & $65 \pm 19 / 75 \pm 20$ \\
FVC $\%$ pred $_{\text {FEV }}$ FVC $\%$ & $81 \pm 15 / 85 \pm 14$ \\
VDP $\%$ & $62 \pm 13 / 68 \pm 13$ \\
$\Delta$ FEV $_{1} / \Delta$ VDP $\mathrm{mL}$ & $10 \pm 8 / 7 \pm 7$ \\
\end{tabular}

\begin{tabular}{lc}
\hline COPD $n=13$ & Pre/PostBD \\
\hline Age years & $70 \pm 6$ \\
Female & $6(46 \%)$ \\
FEV $1 \%$ pred & $47 \pm 12 / 51 \pm 14$ \\
FVC $\%$ pred $_{\text {FEV }}$ FVC $\%$ & $82 \pm 12 / 83 \pm 12$ \\
VDP \% & $43 \pm 9 / 45 \pm 9$ \\
$\Delta$ FEV $_{1} / \Delta V D P ~ m L$ & $24 \pm 9 / 22 \pm 11$ \\
\hline
\end{tabular}

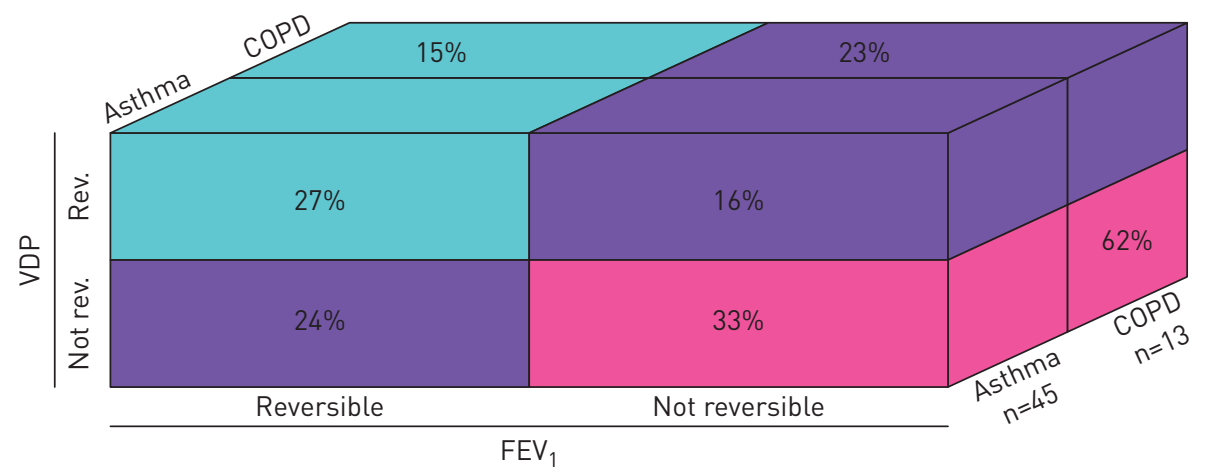

FIGURE 1 Forced expiratory volume in $1 \mathrm{~s}\left(\mathrm{FEV}_{1}\right)$ and magnetic resonance imaging (MRI) ventilation defect per cent (VDP) reversibility in participants with asthma and COPD. Centre slice hyperpolarised ${ }^{3} \mathrm{He}$ MRI static ventilation (blue) co-registered to anatomical proton (greyscale) pre- and post-bronchodilator for representative participants with both $\mathrm{FEV}_{1}$ and MRI VDP reversibility, and with VDP reversibility alone. Tables show demographics for asthma and COPD participants. Cubic illustration shows eight subgroups with proportions of participants in each based on $\mathrm{FEV}_{1}$ and MRI VDP reversibility, where blue represents both $\mathrm{FEV}_{1}$ and VDP reversibility, purple represents either $\mathrm{FEV}_{1}$ or VDP reversibility, and pink represents neither $\mathrm{FEV}_{1}$ nor VDP reversibility. Rev.: reversible.

demonstrated VDP reversibility in the absence of $\mathrm{FEV}_{1}$ reversibility. Overall, VDP was greater in participants with COPD compared with asthma (pre-bronchodilator $24 \pm 9 \%$ versus $10 \pm 8 \%$; $<0.0001$ ), although a similar proportion of participants with COPD and asthma exhibited VDP reversibility (five of 13 or 38\%, COPD; 19 of 45 or $42 \%$, asthma). Figure 1 also shows participant demographics and pre- and post-bronchodilator hyperpolarised ${ }^{3} \mathrm{He}$ static ventilation MRI, for representative participants with both $\mathrm{FEV}_{1}$ and VDP reversibility, and with VDP reversibility alone. There was visually and quantitatively improved ventilation post-bronchodilator even in participants who did not report $\mathrm{FEV}_{1}$ improvements.

We observed a mixture of concordant and discordant FEV $_{1}$ and MRI VDP reversibility in asthmatic and COPD participants and were not surprised that nearly double the proportion of participants with asthma reported concordant $\mathrm{FEV}_{1}$ and VDP reversibility (27\% versus $15 \%$ in COPD). We were surprised that 
nearly the same proportion of asthmatics reported neither $\mathrm{FEV}_{1}$ nor VDP reversibility (33\%), which was about half the proportion of COPD participants (62\%) reporting the same. $\mathrm{FEV}_{1}$ reversibility in asthma may diminish over time, independent of smoking [2], and therefore asthmatics may develop fixed obstruction in a mechanistically different way as compared to COPD patients with post-bronchodilator reversibility $[10,11]$. VDP was previously shown to be an independent predictor of future bronchodilator reversibility in asthmatics [3] and may help identify asthma patients at risk for fixed obstruction over time.

We also observed post-bronchodilator $\mathrm{FEV}_{1}$ and $\mathrm{MRI}$ ventilation improvements in a small number of COPD patients, consistent with previous findings [7]. It is important to note that the COPD participants studied here had no previous history of asthma, and may not meet current asthma-COPD overlap definitions [10, 11]. VDP is temporally persistent and reproducible in COPD [12], so it is possible that corresponding $\mathrm{FEV}_{1}$ and MRI VDP post-bronchodilator improvements identify new phenotypes. Future studies in COPD patients with modest $\mathrm{FEV}_{1}$ and VDP reversibility may show that these patients share similarities with asthma and might benefit from asthma-targeted therapies.

Perhaps most enlightening was the observation in seven asthmatics and three COPD participants who reported reversible VDP in the absence of $\mathrm{FEV}_{1}$ reversibility. This finding lends credence to the growing understanding that $\mathrm{FEV}_{1}$ and VDP are measuring different but complementary pathologies. Given that VDP is more sensitive to small airways disease than $\mathrm{FEV}_{1}$ and VDP predicts future bronchodilator reversibility [3], the lack of VDP reversibility demonstrated in 16\% of the asthma participants studied here may be an early indicator of progression to fixed obstruction [2], which may stem from suboptimal treatment, inflammation, mucus plugging or terminal airway obliteration. We note that two of these seven participants exhibited a post-bronchodilator $\mathrm{FEV}_{1}$ improvement $>200 \mathrm{~mL}$ but did not meet the $12 \%$ threshold $(230 \mathrm{~mL} / 9 \%, 260 \mathrm{~mL} / 11 \%)$ to satisfy American Thoracic Society/European Respiratory Society criteria [8]. The remainder reported post-bronchodilator changes in $\mathrm{FEV}_{1}$ of $-70 \mathrm{~mL} /-4 \%, 160 \mathrm{~mL} / 6 \%$, $-50 \mathrm{~mL} /-5 \%, 130 \mathrm{~mL} / 6 \%$ and $140 \mathrm{~mL} / 10 \%$; some of these post-bronchodilator changes are within the measurement error of $\mathrm{FEV}_{1}$ [8]. Whilst 11 asthmatics reported $\mathrm{FEV}_{1}$ reversibility with no VDP reversibility,

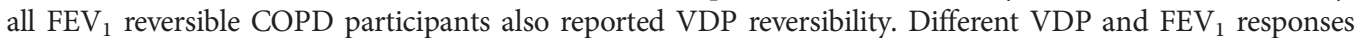
in COPD and asthma may be due to differences in large central airway obstruction and small airways disease that is not detected using spirometry. Previous work showed that MRI in combination with oscillometry revealed that VDP was related to total respiratory system and small airways resistance before bronchodilator inhalation in asthma, but was related only to small airways resistance post-bronchodilator in asthma and COPD [13]. This supports the notion that bronchodilation acts on the large airways in asthma, which could explain why so many participants with asthma but none with COPD showed $\mathrm{FEV}_{1}$ reversibility with no VDP response. These findings challenge us to reflect on the pathophysiological processes related to the distal or peripheral airways which appear to dominate in COPD, but not asthma.

Our current understanding is that there are different asthma and COPD phenotypes with distinctive pathophysiologies, including different contributions of the small and large airways [1]. It was not unexpected that $\mathrm{FEV}_{1}$ and VDP reversibility were not always in agreement, especially because VDP reflects both the small and large airways [14], and $\mathrm{FEV}_{1}$ measurements are dominated by the large airways [15]. Importantly, VDP was not reversible in the majority of participants (34 of 58 total). This is important to consider in phenotypes with small airway disease predominance, in whom reversibility may not be detected using spirometry, and may be considered for inhaled therapy response in general for patients with asthma and COPD. If inhaled mediations are unable to reach the small airways, this could explain why only a subset of patients experience improvements in symptoms or exacerbation frequency. VDP may provide a new way to quantify small and large airway function with enhanced sensitivity to small airways disease and greater dynamic range than $\mathrm{FEV}_{1}$. Our results suggest the possibility that MRI VDP may provide added value for assessing response to inhaled treatments, and perhaps demonstrate different responses for inhalers specifically targeted at the small airways. VDP responses likely depend on patient phenotype and the type of inhaled medication, and this opens the door for future investigations to predict which patients will exhibit a VDP response to which inhaled medication, in an effort to deliver personalised treatment.

We acknowledge that post-bronchodilator response remains controversial in patients with asthma and COPD [11], and this limits the generalisability of our findings. Whilst age and smoking history were clearly different between groups, other parameters considered as key asthma and COPD features [10, 11], including airway inflammation, age of disease-onset and/or disease duration, will be important to consider in future studies.

Post-bronchodilator MRI ventilation defect and $\mathrm{FEV}_{1}$ responses are not always concordant in participants with asthma and COPD, suggesting that VDP may provide a more sensitive tool to measure response to treatment in the small airways and identify the need for a change in treatment. 
Hana Serajeddini ${ }^{1,2,4}$, Rachel L. Eddy ${ }^{1,3,4}$, Christopher Licskai ${ }^{2}$, David G. McCormack ${ }^{2}$ and Grace Parraga ${ }^{1,2,3}$

${ }^{1}$ Robarts Research Institute, Western University, London, ON, Canada. ${ }^{2}$ Division of Respirology, Dept of Medicine, Western University, London, ON, Canada. ${ }^{3}$ Dept of Medical Biophysics, Western University, London, ON, Canada. ${ }^{4}$ Contributed equally as first authors.

Correspondence: Grace Parraga, Robarts Research Institute, 1151 Richmond St N, London, Canada, N6A 5B7. E-mail: gparraga@robarts.ca

Received: 2 Oct 2019 | Accepted after revision: 22 Nov 2019

Conflict of interest: None declared.

Support statement: G. Parraga gratefully acknowledges funding from the Canada Research Chair program, the Canadian Institutes of Health Research and the Natural Sciences and Engineering Research Council (NSERC Canada). R.L. Eddy acknowledges NSERC Canada CGS-Masters and PGS-Doctoral Scholarships.

\section{References}

Postma DS, Rabe KF. The asthma-COPD overlap syndrome. N Engl J Med 2015; 373: 1241-1249.

2 To T, Zhu J, Larsen $\mathrm{K}$, et al. Progression from asthma to chronic obstructive pulmonary disease. Is air pollution a risk factor? Am J Respir Crit Care Med 2016; 194: 429-438.

3 Eddy RL, Svenningsen S, Licskai C, et al. Hyperpolarized helium 3 MRI in mild-to-moderate asthma: prediction of postbronchodilator reversibility. Radiology 2019; 293: 212-220.

4 Kendzerska T, To TM, Aaron SD, et al. The impact of a history of asthma on long-term outcomes of people with newly diagnosed chronic obstructive pulmonary disease: A population study. J Allergy Clin Immunol 2017; 139: 835-843.

5 Sadatsafavi M, Tavakoli H, Kendzerska T, et al. History of asthma in patients with chronic obstructive pulmonary disease. A comparative study of economic burden. Ann Am Thorac Soc 2016; 13: 188-196.

6 Svenningsen S, Kirby M, Starr D, et al. Hyperpolarized (3) He and (129) Xe MRI: differences in asthma before bronchodilation. J Magn Reson Imaging 2013; 38: 1521-1530.

7 Kirby M, Mathew L, Heydarian M, et al. Chronic obstructive pulmonary disease: quantification of bronchodilator effects by using hyperpolarized (3)He MR imaging. Radiology 2011; 261: 283-292.

8 Pellegrino R, Viegi G, Brusasco V, et al. Interpretative strategies for lung function tests. Eur Respir J 2005; 26 : 948-968.

9 Eddy RL, Svenningsen S, McCormack DG, et al. What is the minimal clinically important difference for helium-3 magnetic resonance imaging ventilation defects? Eur Respir J 2018; 51: 1800324.

10 Global Initiative for Obstructive Lung Disease (GOLD) and Global Initiative for Asthma (GINA). Diagnosis of Diseases of Chronic Airflow Limitation: Asthma, COPD and Asthma-COPD Overlap Syndrome (ACOS); 2015. Available from: https://goldcopd.org/asthma-copd-asthma-copd-overlap-syndrome/

11 Sin DD, Miravitlles M, Mannino DM, et al. What is asthma-COPD overlap syndrome? Towards a consensus definition from a round table discussion. Eur Respir J 2016; 48: 664-673.

12 Mathew L, Evans A, Ouriadov A, et al. Hyperpolarized 3He magnetic resonance imaging of chronic obstructive pulmonary disease: reproducibility at 3.0 tesla. Acad Radiol 2008; 15: 1298-1311.

13 Eddy RL, Westcott A, Maksym GN, et al. Oscillometry and pulmonary magnetic resonance imaging in asthma and COPD. Physiol Rep 2019; 7: e13955.

14 Teague WG, Tustison NJ, Altes TA. Ventilation heterogeneity in asthma. J Asthma 2014; 51: 677-684.

15 Macklem PT, Mead J. Resistance of central and peripheral airways measured by a retrograde catheter. J Appl Physiol 1967; 22: 395-401. 